\title{
Polémica en tiempos del COVID-19: ¿es la ivermectina útil para su tratamiento o prevención?
}

\section{Controversy in times of COVID-19: is ivermectin useful for its treat- ment or prevention?}

\author{
Víctor Soto-Cáceres ${ }^{1, a}$
}

\begin{abstract}
En el año 1973, Satoshi Omura del Instituto Kitasato en Japón, y el laboratorio Merck Sharp \& Dohme (MSD) en Estados Unidos de América, iniciaron una colaboración internacional con el fin de producir nuevos fármacos de uso veterinario. En 1975, William Campbell, investigador veterinario americano, evaluó las muestras provenientes de Japón en busca de potenciales agentes terapéuticos de aplicación en la medicina veterinaria. En 1987, la ivermectina fue autorizada para uso humano vía oral como tabletas de $6 \mathrm{mg}$, comenzando así el Programa de Control de la Oncocercosis en países africanos. En 1997, después de la primera década del programa, más de 18 millones de personas estaban siendo tratadas con ivermectina cada año lo que confirmaba la seguridad del fármaco a las dosis indicadas (1).
\end{abstract}

La ivermectina ya casi alcanza los 40 años de empleo y más de 3700 millones de dosis han sido utilizados en el mundo, a más de 68 millones de personas, sin reportarse efectos adversos o fallecimientos entre las personas que lo han tomado y aún se siguen descubriendo indicaciones para su uso en múltiples enfermedades en humanos ${ }^{(2)}$. El profesor Omura y Campbell recibieron el Premio Nobel en Fisiología y Medicina en el 2015.

La ivermectina también ha demostrado gran eficacia en el tratamiento de Demodex folliculorum y $D$. brevis en blefaritis crónica, sin ninguna toxicidad (3). Navarro et al., por su parte, realizaron una revisión de la literatura y un meta análisis sobre la seguridad y dosis de ivermectina concluyendo que las altas dosis son comparables a las dosis estándar en efectos adversos ${ }^{(4)}$. Una revisión publicada en el 2020 destaca la seguridad del producto, e incluso plantea la eficacia del mismo en SARS-CoV-2 ${ }^{(5)}$.

Estudios in vitro también confirmaron que la ivermectina, administrada en concentración de $5 \mu \mathrm{M}$, reducía 5000 veces los niveles de ARN del SARS-CoV-2. Sin embargo, la concentración inhibitoria máxima media (IC50) para el virus era 35 veces mayor que la concentración plasmática máxima. Si se quisiera alcanzar la IC50 a nivel pulmonar, se tendría que utilizar más de 25 veces la dosis aprobada semanalmente. Esto, junto con su efecto antiinflamatorio a nivel local y sistémico, podría soportar su actividad frente a este y otros virus. Su mecanismo de acción antiinflamatorio es desconocido, aunque parece que reduce la producción de algunas interleucinas pro inflamatorias, entre otros eventos.

\section{ESTUDIOS A FAVOR DEL USO DE IVERMECTINA}

Chaccour et al. publicaron en Eclinical Medicine del grupo The Lancet, un pequeño estudio piloto sobre el efecto del tratamiento temprano con ivermectina sobre la carga viral, los síntomas y la respuesta humoral en pacientes con COVID-19 no grave realizando un ensayo clínico piloto, doble ciego, controlado con placebo y aleatorizado. El equipo investigador administró una sola dosis de ivermectina o placebo a 24 pacientes en las 72 horas tras los primeros síntomas. Se tomaron frotis nasofaríngeos y muestras de sangre en el momento del reclutamiento y transcurridas una, dos o tres semanas del tratamiento. "Los resultados obtenidos no muestran ninguna diferencia en el porcentaje de pacientes positivos por PCR al cabo de siete días pos tratamiento (Sin embargo, la carga viral mediana en el grupo tratado con ivermectina fue unas tres veces menor transcurridos cuatro días y hasta 18 veces menor a los siete días del tratamiento). Los pacientes en el grupo tratado también mostraron una reducción en la duración de algunos síntomas (del $50 \%$ en la pérdida del olfato y el gusto y del $30 \%$ en la tos)" (6).

\footnotetext{
1. Universidad de San Martin de Porres, Facultad de Medicina Humana. Chilcayo, Perú

a. Médico especialista en medicina interna y epidemiología, doctor en medicina
} 
En un estudio publicado en octubre 2020, revisado por pares, en Bangladesh, se analizaron retrospectivamente a pacientes con coronavirus en el hospital Mymensingh. De 248 pacientes adultos infectados con SARS-CoV-2, 115 recibieron ivermectina más atención estándar, mientras que 133 solo recibieron atención estándar. El 9,6\% del grupo de ivermectina finalmente requirió la inhalación de oxígeno contra $45,9 \%$ del grupo de atención estándar; el desarrollo de dificultad respiratoria $2,6 \%$ de ivermectina frente al $15,8 \%$ del grupo de atención estándar. Además, de los pacientes que necesitaban tratamiento con antibióticos, 15,7 $\%$ estuvo en el grupo que recibieron ivermectina frente al $60,2 \%$ del grupo de atención estándar. De los pacientes que recibieron ivermectina, 0,9\% terminaron en la unidad de cuidados intensivos ( $\mathrm{UCl}$ ), frente al $8,3 \%$ de los de atención estándar. Aunque los investigadores reconocen que un estudio multicéntrico aleatorizado podría fortalecer la evidencia, tienen claro que la ivermectina debe considerarse como una terapia de primera línea como parte de una estrategia de contención del COVID-19 (7).

En el estudio de cohorte retrospectivo conocido como Ivermectin in Covid Nineteen (ICON), Rajter et al. se plantearon: "determinar si la ivermectina se asocia con una menor tasa de mortalidad en pacientes hospitalizados con COVID-19". Un total de 208 pacientes: 173 tratados con ivermectina y 107 sin ivermectina: disminución de mortalidad en el grupo de ivermectina: $15,0 \%$ vs $25,2 \%$. Mediante ajuste multivariado la diferencia de mortalidad continuaba siendo significativa: $13,3 \%$ frente a $24,5 \%$ (OR: 0,47; IC95\% 0,220,$99 ; p<0,050$ ), con un 11,2\% (IC95\%: 0,38 - 22,1) de reducción de riesgo y con un número necesario a tratar de 8,9 (IC95\%: 4,5 - 263). Concluyendo que el tratamiento con ivermectina estuvo asociado con disminución de mortalidad durante el tratamiento de COVID-19 especialmente en pacientes con daño severo pulmonar ${ }^{(8)}$.

Andrew Hill y el grupo del proyecto internacional sobre ivermectina presentaron el 19 de enero 2021 un meta análisis preliminar de estudios aleatorizados sobre la ivermectina en el tratamiento de la infección por SARS-CoV-2, realizaron una investigación sistemática de PUBMED, EMBASE, MedRXIV y otros registros incluyendo 18 estudios que incluyen 2282 pacientes en la revisión. Los resultados indican que la ivermectina estuvo asociada con la reducción de marcadores inflamatorios: Proteína C Reactiva (PCR), dímero $D$ y ferritina, una rápida disminución viral mediante reacción en cadena de la polimerasa (RT-PCR), y también una reducción significativa de la hospitalización comparada con grupo control. En seis estudios aleatorizados, con pacientes de moderada y severa infección, hubo $75 \%$ de reducción en la mortalidad ( $R R=0,25$; IC 95\%: 0,12-0,52), con una mortalidad de $2,1 \%$ (14/650) en grupo de ivermectina contra $9,5 \%(57 / 597)$ en los controles con una favorable recuperación clínica y disminución del tiempo de hospitalización ${ }^{(9)}$.

Otro estudio de meta análisis en el 2021, indican que: el $100 \%$ de 42 estudios informan efectos positivos, los resultados de mortalidad muestran un $75 \%$ menos de mortalidad, RR 0,25 [0,14-0,44] para todos los retrasos en el tratamiento y un $86 \%$ más bajo, RR 0,14 [0,03 - 0,62] para el tratamiento temprano. El $100 \%$ de los 21 ensayos controlados aleatorios (ECA) informan efectos positivos, con una reducción estimada del $70 \%$, RR 0,30 [0,19$0,49]$ y la probabilidad de que un tratamiento ineficaz genere resultados tan positivos como los 42 estudios hasta la fecha se estima en 1 en 4 billones $(p=0,001)^{(10)}$.

Kory y los investigadores unidos en la FLCCC Alliance indican que la ivermectina tiene un alto potencial antiviral y anti inflamatorio no solo en modelos animales sino también en estudios diversos en humanos tanto en tratamiento como profiláctico y los datos muestran impacto en población mediante "Experimentos naturales que han ocurrido cuando varios países sudamericanos inician distribución de ivermectina mediante campañas a sus pobladores con la esperanza de efectos positivos de esta droga." Existe una asociación temporal de disminución en cantidad de casos y casos fatales en aquellas regiones comparadas con regiones cercanas que no han hecho esas campañas, sugiere que la ivermectina puede probar ser una solución global a la pandemia (11).

En ese sentido, Chamie et al. publicaron un estudio (en imprenta) en Social Science Research Network (SSRN) el 12 de enero 2021, considerando que, "en los nueve departamentos del Perú en los que se distribuyó ivermectina en un corto periodo a través de la operación Tayta, el exceso de muertes disminuyó en $74 \%$ en menos de un mes de haber alcanzado su pico más alto. La caída de mortalidad se inició dentro de los once días del inicio del operativo Tayta ${ }^{(12)}$. Estos detalles fueron publicitados y apoyados por el periodista Gustavo Gorriti a través de IDL-reporteros.

Las guías de tratamiento del COVID-19 de la National Institute Health (NIH) actualizado al 11 de febrero 2021 indica que "los datos son insuficientes para recomendar a favor o en contra del uso de la ivermectina para el tratamiento de COVID-19, se requieren diseños bien conducidos de ensayos clínicos y necesita proveerse de mayor y más específica evidencia sobre el rol de la ivermectina en el tratamiento de COVID-19" (13). En agosto del 2020, el NIH había hecho pública una opinión contraria al uso de la ivermectina.

El 20 de febrero 2021 se difundió que la BIRD (British Ivermectin Recommendation Development) a través de su panel: "recomienda la ivermectina para la prevención y 
tratamiento de COVID-19 para reducir la morbilidad y mortalidad asociada y prevenir la infección COVID-19 entre aquellos de alto riesgo" (14); y Descotes, el 03 de marzo 2021 publica una amplia revisión sobre la seguridad médica de la ivermectina verificando que tiene una amplia seguridad en su uso en los seres humanos ${ }^{(15)}$.

Eslovaquia fue el primer país de la Unión Europea en aprobar el uso de ivermectina tanto como tratamiento como profilaxis por un período de seis meses y el Ministerio de Salud de la República Checa ha autorizado el uso temporal de la ivermectina en los pacientes con COVID-19 el 03 de marzo 2021.

\section{ESTUDIOS EN CONTRA DEL USO DE LA IVERMEC- TINA CONTRA SARS-CoV-2}

Un estudio presentado en octubre 2020 por el Instituto de Evaluación de Tecnologías en Salud e Investigación (IETSI) de la seguridad social EsSalud informaba que "el uso de azitromicina sola, la hidroxicloroquina sola y la ivermectina sola o la combinación de la ivermectina y azitromicina en las primeras 48 horas de hospitalización no mostraría efectos beneficiosos en la disminución de la mortalidad, ingresos a unidades de cuidados intensivos y requerimiento de oxígeno". El artículo fue un pre print presentado por los investigadores Soto-Becerra et al. como "un estudio de cohorte retrospectiva con datos registrados entre abril y julio 2020, con pacientes mayores de 18 años confirmados con SARS-CoV-2 por PCR y que no tenían enfermedad severa al ingreso. El estudio concluye que "no existen efectos beneficiosos de ninguno de los fármacos indicados o sus combinaciones y que al contrario azitromicina + hidroxicloroquina incrementa el riesgo de muerte por todas las causas" (16).

IETSI EsSalud difundió estos datos precisando que, "cuando se usa azitromicina + hidroxicloroquina tiene $84 \%$ más riesgo de fallecer durante hospitalización, $49 \%$ más riesgo de entrar a $\mathrm{UCl}$ y $70 \%$ más riesgo de requerir oxígeno" (17). En los medios de comunicación se difundió unas de sus conclusiones: "que aumentaría en $84 \%$ el riesgo de muerte entre los pacientes hospitalizados por COVID-19" y el gerente general de EsSalud y los expertos como el Dr. Eduardo Gotuzzo indicaron que "estaba muy claro que son medicamentos que no se deben emplear en pacientes hospitalizados, pero sí es manejable en pacientes ambulatorios y pacientes de riesgo", siendo esa conclusión de que las personas tienen mayor riesgo de morir incorrecta, ya que es una revisión de historias que pretende ser un ensayo clínico cuando no lo es.

Un reciente estudio en imprenta de autores peruanos, revisan cinco bases de datos y páginas web por pre prints que fueron remitidos hasta enero 2021 sobre estudios clínicos controlados aleatorizados y cohortes retrospectivas sobre el uso de ivermectina versus control en pacientes ambulatorios y hospitalizados; utilizando como puntos finales mortalidad y recuperación de pacientes. En sus resultados refieren que "doce estudios fueron incluidos (cinco cohortes retrospectivas, seis ensayos clínicos aleatorizados y una serie de casos) con un total de 7412 pacientes con edad mediana de 47,5 años y $58 \%$ fueron varones. La ivermectina no fue asociada con reducción de mortalidad $\left(\operatorname{logRR}=0,89 ; C|95=0,09-1,70 ; p=0,040 ;|^{2}=84,7 \%\right)$, o reducción en recuperación del paciente $(\operatorname{logRR}=5,52$; Cl95\% $=-24.36-35,4 ; p=0,510 ;\left.\right|^{2}=92,6 \%$ ), indicando que todos los estudios tienen alto riesgo de error y mostraron baja certeza de evidencia, concluyendo que existe insuficiente certeza y calidad de evidencia para recomendar el uso de ivermectina para prevención o tratamiento en pacientes ambulatorios u hospitalizados con COVID-19" (18).

Datos del panel de IDSA (Sociedad Americana de Enfermedades Infecciosas) dan una recomendación 18: "en pacientes hospitalizados con severo COVID-19 sugiere en contra del uso de ivermectina por muy baja certeza de evidencia" y la recomendación 19 en pacientes no hospitalizados el panel sugiere en contra de la ivermectina fuera del contexto de un ensayo clínico por muy baja certeza de evidencia" (19).

López-Medina publica el 04 de marzo 2021 en JAMA un ensayo clínico aleatorizado con el objetivo de determinar si la ivermectina es un tratamiento eficaz para COVID-19 leve, conduciendo un estudio doble ciego, aleatorizado, en Cali Colombia, en pacientes confirmados con enfermedad leve y sintomáticos. Un grupo de pacientes recibieron ivermectina diaria por cinco días y otro grupo recibió placebo, la mediana de resolución de síntomas fue de 10 días (IC95\%=9-13) comparado con 12 días en grupo placebo $(I C 95 \%=9-13)$. Concluyeron que la administración por cinco días de ivermectina no disminuye significativamente el tiempo de resolución de síntomas siendo necesario mayores estudios ${ }^{(20)}$.

\section{COMENTARIOS FINALES}

El uso de la ivermectina ha recibido opiniones favorables y críticas en contra de su uso en la pandemia del COVID-19 aduciendo no haberse demostrado mediante "evidencias" su utilidad real. Sin embargo, la evidencia científica clínica aparece desde los estudios descriptivos transversales en los cuales se establece asociación estadística entre posibles factores y eventos (enfermedades) planteándose hipótesis concretas que luego los estudios analíticos de casos y controles y posteriormente los de cohortes establecen los factores de riesgo apuntando hacia la causalidad de la enfermedad. Pero también en la definición de aquellos factores protectores que evitan que uno se complique en una determinada situación, no son 
solo evidencia los ensayos clinicos controlados pues incluso encima de ellos están los denominados meta análisis.

La ivermectina es una droga con seguridad comprobada a lo largo del tiempo y en el peor de los escenarios si no tuviera efectos beneficiosos tampoco ocasiona daño a las dosis estandarizadas de uso frecuente y en el mejor de los escenarios si tuviera real actividad impediría el avance hasta serias complicaciones en un paciente infectado por el SARS-CoV-2 por lo que, a criterio personal, constituye una alternativa ante la otra situación de dar únicamente sintomáticos para dolor o fiebre a un paciente que inicia molestias y esperar la evolución natural de la enfermedad que puede desencadenar en complicaciones graves incluyendo el fallecimiento.

Fuentes de financiamiento: Autofinanciado.

Conflicto de intereses: No hay conflicto de intereses

\section{REFERENCIAS BIBLIOGRÁFICAS}

1. Omura S, Crump A. The life and times of ivermectin - a success story. Nat Rev Microbiol. 2004; 2(12): 984-9. doi: 10.1038/nrmicro1048.

2. Victoria Ch J. Ivermectina: sus múltiples usos, seguridad y toxicidad. Rev Chil Dermatol. 2010; 26(4): 358-68. Disponible en: https://www.sochiderm.org/web/revista/26_4/1.pdf

3. Czepita D, Kuzna-Grygiel W, Czepita M, Grobelny A. Demodex folliculorum and Demodex brevis as a cause of chronic marginal blepharitis. Ann Acad Med Stetin. 2007;53(1):63-7; discussion 67. Ann Acad Med Stetin. 2007;53(1):63-7; discussion 67. Disponible en: https://pubmed.ncbi.nlm. nih.gov/18561612/

4. Navarro M, Camprubì D, Requena-Méndez A, Buonfrate D, Giorli G, Kamgno J et al. Safety of high-dose ivermectin: a systematic review and meta-analysis.J Antimicrob Chemother Enero 2020. doi:10.1093/jac/ dkz524

5. Luque -Espino JC, Pareja C A. Seguridad y eficacia de ivermectina en tiempos de COVID-19. Horiz Med (Lima) 2020; 20(4):e1393. https://doi. org/10.24265/horizmed.2021.v20n1.2

6. Chaccour C, Casellas A, Blanco-Di Matteo A, Pineda I, Fernández-Montero A, Ruiz-Castillo P. et al., The effect of early treatment with ivermectin on viral load, symptoms and humoral response in patients with non-severe COVID-19: A pilot, double-blind, placebo-controlled, randomized clinical trial, E Clinical Medicine. 2021; 30, 100720. https://doi.org/10.1016/j. eclinm.2020.100720

7. Khan SI, Khan SI, Debnath CR, Nath PN, Mahtab MA, Nabeka H, Matsuda S. Ivermectin Treatment May Improve the Prognosis of Patients With COVID-19. Arch Bronconeumol. 2020 october 24;56 (12):816-830. https://doi.org/10.1016/j.arbres.2020.08.0070300-2896/

8. Rajter JC, Sherman MS, Faltteh N, Vogel F Sacks J, Raiter JJ. Use of Ivermectin Is Associated With Lower Mortality in Hospitalized Patients With Coronavirus Disease 2019. The Ivermectin in COVID Nineteen Study. CHEST 2021; 159(1):85-92. https://doi.org/10.1016/j.chest.2020.10.009

9. Hill A, Abdulamir AS, Ahmed S, Asghar A, Babalola OE, Basri R et al. Meta-analysis of randomized trials of ivermectin to treat SARS-CoV-2 infection. Research Square. DOI: 10.21203/rs.3.rs-148845/v1

10. Covid Analysis. La ivermectina es eficaz para COVID-19: metanálisis en tiempo real de 42 estudios [Internet]. 2020 [citado el 24 de febrero de 2021]. Disponible en: https://ivmmeta.com/

11. Kory P, Meduri U, Iglesias J, Varon J, Berkowitz K, Kornfeld H, et al. Review of the Emerging Evidence Demonstrating the Efficacy of Ivermec- tin in the Prophylaxis and Treatment of COVID-19 [Internet]. FLCCC Alliance updated. 2021 [citado el 12 de enero de 2021]. Disponible en: https://osf.io/wx3zn/?fbclid=IwAR3eqil6nDsaD96QRDosGgjPF9JO_fbz1BVkiL8Guiibzlfdc_LT156INYI

12. Chamie-Quintero J, Hibberd J, Scheim D. Sharp Reductions in COVID-19 Case Fatalities and Excess Deaths in Peru in Close Time Conjunction, State-By-State, with Ivermectin Treatments (January 12, 2021). http://dx.doi.org/10.2139/ssrn.3765018

13. NIH COVID-19 Treatment Guidelines. Ivermectin [Internet]. Last Updated: February 11, 2021. Disponible en: https://www.covid19treatmentguidelines.nih.gov/antiviral-therapy/ivermectin/

14. BIRD The BIRD Recommendation on the use of Ivermectin for Covid-19. Executive Summary [Internet]. Meeting in Bath UK 20 february 2021. Disponible en: https://www.francesoir.fr/sites/francesoir/files/media-icons/bird-proceedings-02-03-2021-v151.pdf

15. Descotes J. Expert Review Report Medical Safety of Ivermectin [Internet]. 03 marzo 2021. Disponible: https://www.bloomberg.com/press-releases/2021-03-05/covid-19-medincell-publishes- an-extensive-ivermectin-safety-expert-analysis

16. Soto-Becerra P, Culquichicón C, Hurtado-Roca Y, Araujo-CastiIlo RV. Real-world effectiveness of hydroxychloroquine, azithromycin, and ivermectin among hospitalized COVID-19 patients: results of a target trial emulation using observational data from a nationwide healthcare system in Peru. medRxiv preprint. 2020. doi: https://doi. org/10.1101/2020.10.06.20208066

17. IETSI. Desenlaces en pacientes hospitalizados con COVID-19 en EsSalud [Internet]. 08 octubre 2020. Disponible en: www.essalud.gob.pe

18. Castañeda-Sabogal A, Chambergo-Michilot $D$, Toro-Huamanchumo $C J$, Silva-Rengifo C, Gonzales-Zamora J, Barboza JJ. Outcomes of Ivermectin in the treatment of COVID-19: a systematic review and meta-analysis. medRxiv pre print doi: https://doi.org/10.1101/2021.01.26.21250420

19. IDSA. Recommendations 18-19: Ivermectin vs no ivermectin for hospitalized patients and outpatients outside the context of a clinical trial [Internet]. Section last reviewed and updated 2/13/2021. Disponible en: https://www.idsociety.org/practice-guideline/covid-19-guideline-treatment-and-management/

20. López-Medina E, López P, Hurtado I, Dávalos DM, Ramirez O, Martínez E, Díaz- Granados JA et al. Effect of Ivermectin on Time to Resolution of Symptoms Among Adults With Mild COVID-19. A Randomized Clinical Trial. JAMA. Published online March 4, 2021. doi:10.1001/jama.2 